Proyecciones

Vol. 23, No 2, pp. 123-129, August 2004.

Universidad Católica del Norte

Antofagasta - Chile

\title{
SOLVABILITY OF COMMUTATIVE POWER-ASSOCIATIVE NILALGEBRAS OF NILINDEX 4 AND DIMENSION
}

\author{
LUISA ELGUETA \\ and \\ AVELINO SUAZO \\ Universidad de La Serena, Chile \\ Received : October 2003. Accepted : May 2004
}

\begin{abstract}
Let $A$ be a commutative power-associative nilalgebra. In this paper we prove that when $A$ (of characteristic $\neq 2$ ) is of dimension $\leq 8$ and $x^{4}=0$ for all $x \in A$, then $\left(\left(A^{2}\right)^{2}\right)^{2}=0$. That is, $A$ is solvable. We conclude that if $A$ is of dimension $\leq 7$ over a field of characteristic $\neq 2,3$ and 5 , then $A$ is solvable.
\end{abstract}




\section{PRELIMINARIES}

Let $A$ be a commutative algebra over a field $K$. If $x$ is an element of $A$, we define $x^{1}=x$ and $x^{k+1}=x^{k} x$ for all $k \geq 1$. $A$ is called power-associative, if the subalgebra of $A$ generated by any element $x \in A$ is associative. An element $x \in A$ is called nilpotent, if there is an integer $r \geq 1$ such that $x^{r}=0$. If any element in $A$ is nilpotent, then $A$ is called a nilalgebra. Now $A$ is called a nilalgebra of nilindex $n \geq 2$, if $y^{n}=0$ for all $y \in A$ and there is $x \in A$ such that $x^{n-1} \neq 0$.

If $B, D$ are subspaces of $A$, then $B D$ is the subspace of $A$ spanned by all products $b d$ with $b$ in $B, d$ in $D$. Also we define $B^{1}=B$ and $B^{k+1}=B^{k} B$ for all $k \geq 1$. If there exists an integer $n \geq 2$ such that $B^{n}=0$ and $B^{n-1} \neq 0$, then $B$ is nilpotent of index $n$.

$A$ is called solvable in case $A^{(k)}=0$ for some integer $k$, where $A^{(1)}=A$ and $A^{(n+1)}=\left(A^{(n)}\right)^{2}$ for all $n \geq 1$.

$A$ is a Jordan algebra, if it satisfies the Jordan identity $x^{2}(y x)=\left(x^{2} y\right) x$ for all $x, y$ in $A$, and $A$ is a commutative algebra. It is known that any Jordan algebra (of characteristic $\neq 2$ ) is power-associative, and also that any finite-dimensional Jordan nilalgebra is nilpotent (see, [7]).

It is known that if $A$ is a commutative algebra such that the identity $x^{3}=0$ is valid in $A$, then $A$ is a Jordan algebra (see [9], page 114). Therefore, if $A$ is a finite dimensional algebra then $A$ is nilpotente, and hence solvable.

We will denote by $\left\langle a_{1}, \ldots, a_{j}>K\right.$ the subspace of $A$ generated over $K$ by the elements $a_{1}, \ldots, a_{j} \in A$. In the following a greek letter indicates an element of the field $K$.

In [8], D. Suttles constructs (as a counterexample to a conjecture due to A. A. Albert) a commutative power-associative nilalgebra of nilindex 4 and dimension 5, which is solvable and is not nilpotent. In [3] (Theorem 3.3), we prove that this algebra is the unique commutative power-associative nilalgebra of nilindex 4 and dimension 5, which is not Jordan algebra. At present there exists the following conjecture: Any finite-dimensional commutative power-associative algebra is solvable. The solvability of these algebras for dimension 4, 5 and 6 , are proved in [5], [3] and [2] respectively.

From Theorem 2 of [4] and [6] we obtain the following result:

Theorem 1.1 : If $A$ is a commutative power-associative nilalgebra of nilindex $n$ with dimension $\leq n+2$ and the characteristic is zero or $\geq n$, 
then $A$ is solvable.

The following result is proved in [3] :

Lemma 1.2 : If $A$ is a commutative power-associative nilalgebra of nilindex 4 , dimension 5 and is not a Jordan algebra, then $\operatorname{dim}_{K}\left(A^{2}\right)=3$.

\section{SOLVABILITY}

In this section, $A$ is a commutative power-associative algebra over a field $K$ with characteristic $\neq 2$, such that the identity $x^{4}=0$ is valid in $A$. Linearizing the identities $\left(x^{2}\right)^{2}=0$ and $x^{4}=0$, we obtain that for all $y, x, z, v$ in $A$ :

$$
\begin{gathered}
(y x) x^{2}=0,2(x y)^{2}+x^{2} y^{2}=0 \\
x^{2}(y z)+2(x y)(x z)=0,\left(x^{2} y\right)\left(x^{2} v\right)=0 \\
(x y)(z v)+(x z)(y v)+(x v)(y z)=0 \\
2((y x) x) x+\left(y x^{2}\right) x+y x^{3}=0
\end{gathered}
$$

We see that replacing $y$ by $y x$ in (4) and using (1), we obtain that $2(((y x) x) x) x+(y x) x^{3}=0$. Replacing $z$ by $x^{2}$ in (2), we get $x^{2}\left(y x^{2}\right)+$ $2(y x) x^{3}=0$. Therefore for all $y, x$ in $A$ we have that:

$$
4(((y x) x) x) x=x^{2}\left(y x^{2}\right)=-2(y x) x^{3}
$$

If now we replace $y$ by $y x$ in (5) and using (1), we obtain that:

$$
((((y x) x) x) x) x=0
$$

We observe that using the identity (6), it is easy to prove the following result:

Lemma 2.1 :If $y, x$ are elements in $A$ such that $y \neq 0$ and $x y=\alpha y$, then $\alpha=0$.

Lemma 2.2 : If $A$ is of dimension $\leq 8$, then the following identities are valid in $A$ :

$$
\begin{gathered}
(((y x) x) x) x=x^{2}\left(y x^{2}\right)=(y x) x^{3}=0 \\
(y x)^{3}=0
\end{gathered}
$$


Proof. We will prove first that $(((y x) x) x) x=0$ for all $y, x$ in $A$. Suppose that there exist elements $y, x$ in $A$ such that $(((y x) x) x) x \neq 0$. By $(5)$ we have that $x^{2}\left(y x^{2}\right) \neq 0$ and $x^{3} \neq 0$. Let $X=<x, x^{2}, x^{3}>_{K}$. The elements $x^{2}\left(y x^{2}\right)$ and $x^{3}$ are linearly independent. In fact: If $x^{2}\left(y x^{2}\right)=\sigma x^{3}$, then by (5) we get that $(y x) x^{3}=-\frac{1}{2} \sigma x^{3}$. Using the Lemma 2.1, we obtain that $\sigma=0$, which is a contradiction. We note that we can have that either $(((y x) x) x) x \in X$ or $(((y x) x) x) x \notin X$. Suppose that $(((y x) x) x) x \notin X$. In this case we will prove that $y, y x,(y x) x,((y x) x) x,(((y x) x) x) x, x, x^{2}$, $x^{3}, y x^{2}$ are linearly independent. Let $\alpha y+\beta y x+\gamma(y x) x+\delta((y x) x) x+$ $\varepsilon(((y x) x) x) x+\beta_{1} x+\beta_{2} x^{2}+\beta_{3} x^{3}+\lambda y x^{2}=0$. Multiplying by $x^{2}$ and using (1), we get that $\alpha y x^{2}+\beta_{1} x^{3}+\lambda\left(y x^{2}\right) x^{2}=0$. Now using (5) and (1) : $0=x^{2}\left(\alpha y x^{2}+\beta_{1} x^{3}+\lambda\left(y x^{2}\right) x^{2}\right)=\alpha x^{2}\left(y x^{2}\right)$ implies $\alpha=0$, and since $x^{3}$, $\left(y x^{2}\right) x^{2}$ are linearly independent, we get $\beta_{1}=\lambda=0$. Hence we have that $\beta y x+\gamma(y x) x+\delta((y x) x) x+\varepsilon(((y x) x) x) x+\beta_{2} x^{2}+\beta_{3} x^{3}=0$. Multiplying two times by $x$ and using (6), we get that $\beta=\gamma=0$. Multiplying by $x$ we get that $0=\delta(((y x) x) x) x+\beta_{2} x^{3}=\frac{1}{4} \delta x^{2}\left(y x^{2}\right)+\beta_{2} x^{3}$, which implies $\delta=\beta_{2}=0$. Now $0=\varepsilon(((y x) x) x) x+\beta_{3} x^{3}=\frac{1}{4} \varepsilon x^{2}\left(y x^{2}\right)+\beta_{3} x^{3}$ implies $\varepsilon=\beta_{3}=0$. Thus we conclude that $\operatorname{dim}_{K}(A) \geq 9$, which is a contradiction. Therefore we must have $(((y x) x) x) x \in X$, and so $(((y x) x) x) x=\alpha_{1} x+\alpha_{2} x^{2}+\alpha_{3} x^{3}$. Now $0=((((y x) x) x) x) x=\alpha_{1} x^{2}+\alpha_{2} x^{3}$ implies $\alpha_{1}=\alpha_{2}=0$, and relation (5) together with Lemma 2.1 imply $\alpha_{3}=0$. That is, $(((y x) x) x) x=0$, a contradiction. Hence we prove that the identity $(((y x) x) x) x=0$ is valid in $A$, and thus by (5) we obtain (7).

Now we will prove the identity (8). We know that $x^{2}\left(y x^{2}\right)=0$ for all $y$, $x$ in $A$. By linearization we get that the following identity is valid in $A$ :

$$
x^{2}((x z) y)+(x z)\left(y x^{2}\right)=0
$$

Replacing $z$ by $x^{3}$ in (2) we get $x^{2}\left(y x^{3}\right)=0$, and replacing $z$ by $x^{2}$ in (9) we obtain $x^{3}\left(y x^{2}\right)=-x^{2}\left(y x^{3}\right)=0$. Finally, replacing $z$ by $y, y$ by $y^{2}$ in (9) and using (1), we obtain that $0=x^{2}\left((x y) y^{2}\right)=-(x y)\left(y^{2} x^{2}\right)=2(y x)^{3}=0$.

Remark 2.3 : Lemma 2.2 is not valid when $A$ is of dimension 9 . In fact : Let $B$ be a commutative algebra of dimension 9 with basis $\left\{v_{1}, v_{2}, v_{3}, v_{4}, v_{5}, v_{6}, v_{7}, v_{8}, v_{9}\right\}$ and nonzero multiplication given by $: v_{1} v_{6}=$ $v_{2}, v_{1} v_{7}=v_{9}, v_{1} v_{8}=-(2+\beta) v_{4}-\gamma v_{5}, v_{2} v_{6}=v_{3}, v_{2} v_{8}=-2 v_{5}, v_{3} v_{6}=v_{4}$, $v_{4} v_{6}=v_{5}, v_{6}^{2}=v_{7}, v_{6} v_{7}=v_{8}, v_{6} v_{9}=\beta v_{4}+\gamma v_{5}, v_{7} v_{9}=4 v_{5}$. We obtain that $B$ is a commutative power-associative nilalgebra of nilindex 4 , such that: $\left(\left(\left(v_{1} v_{6}\right) v_{6}\right) v_{6}\right) v_{6}=v_{5},\left(v_{1} v_{6}^{2}\right) v_{6}^{2}=4 v_{5}$ and $\left(v_{1} v_{6}\right) v_{6}^{3}=-2 v_{5}$. 
Theorem 2.4 : If $A$ is of dimension $\leq 8$, then $\left(\left(A^{2}\right)^{2}\right)^{2}=0$.

Proof. Suppose that $\left(\left(A^{2}\right)^{2}\right)^{2} \neq 0$. Then there exist elements $y, x, u, v$ in $A$ such that $\left(x^{2} y^{2}\right)\left(u^{2} v^{2}\right) \neq 0$. From (1) we obtain that $\left(x^{2} y^{2}\right)\left(u^{2} v^{2}\right)=$ $4(x y)^{2}(u v)^{2}=-8((x y)(u v))^{2} \neq 0$. We will prove that $x^{2}, y^{2}, x y, x^{2} y^{2}$, $u^{2}, v^{2}, u v, u^{2} v^{2}$ are linearly independent. The elements $x^{2}, y^{2}, x y, x^{2} y^{2}$ are linearly independent. In fact: if $\alpha x^{2}+\beta y^{2}+\gamma x y+\delta x^{2} y^{2}=0$, then multiplying by $x^{2}$ and using the identities (1) and (7) we obtain that $\beta x^{2} y^{2}=0$, which implies $\beta=0$. Similarly we obtain that $\alpha=0$. Now using (8), $0=\gamma y x+\delta x^{2} y^{2}=\gamma y x-2 \delta(x y)^{2}$ implies $\gamma=\delta=0$. Let $\alpha x^{2}+\beta y^{2}+\gamma x y+\delta x^{2} y^{2}+\alpha_{0} u^{2}+\beta_{0} v^{2}+\gamma_{0} u v+\delta_{0} u^{2} v^{2}=0$. Multiplying by $v^{2}$, afterwards by $x^{2} y^{2}=-2(x y)^{2}$ and using (1), (2) and (7) we obtain $\alpha_{0}\left(x^{2} y^{2}\right)\left(u^{2} v^{2}\right)=0$, which implies $\alpha_{0}=0$. Similarly, $\beta_{0}=0$. Now we have that $\alpha x^{2}+\beta y^{2}+\gamma x y+\delta x^{2} y^{2}=-\left(\gamma_{0} u v+\delta_{0} u^{2} v^{2}\right)$. Using (1), (7) and (8): $\left(\alpha x^{2}+\beta y^{2}+\gamma x y+\delta x^{2} y^{2}\right)^{2}=\left(2 \alpha \beta-\frac{1}{2} \gamma^{2}\right) x^{2} y^{2}$ and $\left(-\left(\gamma_{0} u v+\delta_{0} u^{2} v^{2}\right)\right)^{2}=-\frac{1}{2} \gamma_{0}^{2} u^{2} v^{2}$. Hence $\left(2 \alpha \beta-\frac{1}{2} \gamma^{2}\right) x^{2} y^{2}=-\frac{1}{2} \gamma_{0}^{2} u^{2} v^{2}$, which implies that $-\frac{1}{2} \gamma_{0}^{2}\left(u^{2} v^{2}\right)\left(x^{2} y^{2}\right)=0$, and so $\gamma_{0}=0$. Now we have that $\alpha x^{2}+\beta y^{2}+\gamma x y+\delta x^{2} y^{2}+\delta_{0} u^{2} v^{2}=0$. Multiplying by $x^{2} y^{2}$ we get $\delta_{0}=0$. Since $x^{2}, y^{2}, x y, x^{2} y^{2}$ are linearly independent, we conclude that $\left\{x^{2}, y^{2}, x y, x^{2} y^{2}, u^{2}, v^{2}, u v, u^{2} v^{2}\right\}$ is a basis of $A$, and hence $A^{2}=A$. Now using the above identities we get that $A=A^{2}=<x^{2} y^{2}, u^{2} v^{2}, x^{2} u^{2}, x^{2} v^{2}$, $x^{2}(u v), x^{2}\left(u^{2} v^{2}\right), y^{2} u^{2}, y^{2} v^{2}, y^{2}(u v), y^{2}\left(u^{2} v^{2}\right),(x y) u^{2},(x y) v^{2},(x y)(u v)$, $(x y)\left(u^{2} v^{2}\right),\left(x^{2} y^{2}\right) u^{2},\left(x^{2} y^{2}\right) v^{2},\left(x^{2} y^{2}\right)(u v),\left(x^{2} y^{2}\right)\left(u^{2} v^{2}\right)>_{K}$. Let $J=<$ $\left(x^{2} y^{2}\right)\left(u^{2} v^{2}\right)>_{K}$. We observe that $\left(x^{2} y^{2}\right) A=J$ and $\left(u^{2} v^{2}\right) A=J$. Now we will show that $\left(\left(x^{2} y^{2}\right)\left(u^{2} v^{2}\right)\right) A=0$. Since $A^{2}=A$, it is sufficient to prove that $\left(\left(x^{2} y^{2}\right)\left(u^{2} v^{2}\right)\right)\left(z_{1} z_{2}\right)=0$ for all $z_{1}, z_{2} \in A$. Now using (3), we obtain that $\left(\left(x^{2} y^{2}\right)\left(u^{2} v^{2}\right)\right)\left(z_{1} z_{2}\right)=-\left(\left(x^{2} y^{2}\right) z_{1}\right)\left(\left(u^{2} v^{2}\right) z_{2}\right)-\left(\left(x^{2} y^{2}\right) z_{2}\right)\left(\left(u^{2} v^{2}\right) z_{1}\right) \in$ $J^{2}=0$, and therefore $\left(\left(x^{2} y^{2}\right)\left(u^{2} v^{2}\right)\right) A=0$. It is easy to prove that $x^{2} y^{2}$, $u^{2} v^{2},\left(x^{2} y^{2}\right)\left(u^{2} v^{2}\right)$ are linearly independent, and moreover clearly the subspace $I=<x^{2} y^{2}, u^{2} v^{2},\left(x^{2} y^{2}\right)\left(u^{2} v^{2}\right)>_{K}$ is an ideal of $A$. Now $\bar{A}=A / I$ is a commutative power-associative nilalgebra of dimension 5 . If $\bar{A}$ is a Jordan algebra, then is nilpotent and so $\operatorname{dim}_{K}\left(\bar{A}^{2}\right)<5$. Now if $\bar{A}$ is not a Jordan algebra, then by Lemma 1.2 we get that $\operatorname{dim}_{K}\left(\bar{A}^{2}\right)<5$. Finally we conclude that $\bar{A}^{2}=A^{2} / I=A / I=\bar{A}$, which is a contradiction. Therefore $\left(\left(A^{2}\right)^{2}\right)^{2}=0$, as desired.

Finally, by Theorems 1.1 and 2.4 we get:

Corollary 2.5 : Let $A$ be a commutative power-associative nilalgebra over a field of characteristic $\neq 2$,3and 5 .If $A$ is of dimension $\leq 7$, then $A$ is solvable. 
The authors thank the referee for many useful remarks.

\section{REFERENCES}

[1] Correa, I.; Suazo, A. On a class of commutative power-associative nilalgebras. Journal of Algebra, 215, pp. 412-417, (1999).

[2] Correa, I.; Hentzel I. R.; Peresi, L. A. On the solvability of the commutative power-associative nilalgebras of dimension 6. Linear Alg. Appl., 369, pp. 185-192, (2003).

[3] Elgueta, L.; Suazo, A. Jordan nilalgebras of nilindex $n$ and dimension $n+1$. Communications in Algebra, 30, pp. 5547-5561, (2002).

[4] Elgueta, L.; Gutierrez Fernandez, J. C.; Suazo, A. Nilpotence of a class of commutative power-associative nilalgebras. Submitted.

[5] Gerstenhaber, M.; Myung, H.C. On commutative power-associative nilalgebras of low dimension. Proc. Amer. Math. Soc., 48, pp. 29-32, (1975).

[6] Gutierrez Fernandez J.C. On commutative power-associative nilalgebras. Communications in Algebra, 32(6), pp. 2243-2250, (2004).

[7] Schafer, R.D. An Introduction to Nonassociative Algebras; Academic Press: New York/London, (1966).

[8] Suttles, D.A. Counterexample to a conjeture of Albert. Notices Amer. Math. Soc. , 19, A-566, (1972).

[9] Zhevlakov, K.A.; Slin'ko, A.M.; Shestakov, I.P.; Shirshov, A.I. Rings That Are Nearly Associative; Academic Press: New York/London, (1992). 


\section{Luisa Elgueta}

Departamento de Matemáticas

Universidad de La Serena

Cisternas 1200

La Serena

Chile

e-mail : lelgueta@userena.cl

and

\section{Avelino Suazo}

Departamento de Matemáticas Universidad de La Serena

Cisternas 1200

La Serena

Chile

e-mail : asuazo@userena.cl 